\title{
Title: CHRONIC PIPELINE LEAK DETECTION SURVEY: A DESCRIPTION OF VARIOUS CASE STUDIES
}

\author{
Niresh Behari P.Eng', Dr Azizur Rahman² \\ ABSTRACT \\ ${ }^{1}$ Memorial University of Canada Faculty of Chemical Process Engineering and Process Safety Expert in \\ Shell Canada \\ ${ }^{2}$ Texas A\&M University at Qatar Faculty of Chemical Process Engineering
}

A pipeline leak detection survey for the oil and gas sector is investigated with aim of determining accuracy, resource level requirements and risk of installation and operation for various technologies. Medium to large scale leaks between 3 to $10 \mathrm{~mm}$ could be detected using dynamic pressure wave monitoring for single phase flow whereas sequential probability ratio testing (SPRT) using real time transient monitoring (RTTM) can be used to monitor leaks for multiphase flows even in offshore shallow water conditions and along elevated pipeline networks. Chronic leaks could be measured based on accuracy of hydrocarbon and pressure monitoring devices installed in a vacuum annulus pipe-in-pipe arrangement however the pipeline length is restricted due to weight and difficulty of pipeline installation. Distributed temperature sensing and distributed acoustic sensing leak detection using fiber optic cable (FOC) were found to be resource intensive and have higher installation and operation risks due to unknown equipment reliability and location or sealing of the FOC on the pipeline structure. RTTM and SPRT have comparable accuracy to DTS or DAS leak detection and can be retrofitted to existing pipeline networks. None of the leak detection technologies evaluated had proven capability to predict leaks for pipelines manufactured from reinforced thermoplastic (RTP) materials.

Keywords Leak Detection System, Real Time Transient Monitoring, Reinforced Thermoplastics

\section{INTRODUCTION}

Chronic or small pipeline leaks can be a significant contributor to GHG emissions resulting in global warming and environmental pollution. Pipeline and gas facility processing leaks account for $23 \%$ of GHG emissions in Canada [1]. If these leaks go unattended within prescribed time limits they can deteriorate and may cause large scale environmental release, fires or explosions. There is currently dissension in petrochemical industries about the time duration to repair onshore chronic pipeline leaks since any leak below 100,000ppm measured at the source is usually not repaired until the next planned shutdown or a subsequent leak measurement is recommended 3 years later as part of a leak detection and repair (LDAR) study. Usually for chronic hydrocarbon leaks greater than 100,000ppm a risk based approach is used to repair the leak or reduce the leak flow rate however for offshore deep water or arctic conditions chronic leaks need to be repaired in the shortest time possible due to the large scale environmental impact and potential for the leak to escalate to a catastrophic rupture [2].

The benefits of this study provide the functionality and error limitations of recent leak detection and location technologies together with their pitfalls based on published field or experimental data. Leak detection calibration tables describing flow properties, pressure and flow monitoring for various technologies are provided and supported with computational fluid dynamic (CFD) modelling of the leak dispersion for simple pipeline arrangements in shallow water and onshore conditions. Moreover comparative charts are provided to illustrate the increasing project and installation cost, operations risk, chronic leak size and accuracy limitations.

Developing and implementing a pipeline repair strategy for subsea conditions more than $3000 \mathrm{~m}$ deep or in sub zero temperature arctic conditions near ice formations can be challenging. It is therefore critical to 
detect small hydrocarbon leaks in the shortest time possible using effective leak detection systems (LDS). Some of the recent LDS technologies used for metallic pipeline leaks include:

- Negative or Dynamic Pressure Wave technology with effective signal to noise ratio (SNR)

- $\quad$ Real Time Transient Monitoring (RTTM) with Sequential Probability Ratio Testing(SPRT)

- Distributed Temperature Sensing (DTS) or Distributed Acoustic Sensing (DAS) using Fiber Optic Cable and Brillouin light scattering

- Vacuum pressure annulus monitoring

There are three types of fiber optic cable leak detection and location technologies namely Distributed Strain Sensing (DSS), DTS and DAS that are used in pilot experiments and new pipelines compared to negative or dynamic pressure wave that relies on customized SNR filters, which characterize the type of flow, environmental conditions and pipe damping properties. SPRT and RTTM is used for complex pipeline networks for any type of flow conditions and is suited for onshore and offshore applications. Large or catastrophic leaks greater than $10 \mathrm{~mm}$ can be detected using Dynamic pressure sensing, whereas medium to small leaks between 4 to $8 \mathrm{~mm}$ is suited to RTTM and SPRT lead detection and location technology. Pipeline leaks greater than $4 \mathrm{~mm}$ for onshore, arctic and subsea conditions were investigated however there is no published field data available which fully addresses chronic leaks less than 3mm [3-5]. Experimental and pilot scale studies using fiber optic leak detection highlighted key risks using DTS or DAS for arctic or subsea conditions [4]. The literature shows that no field studies describing complex pipeline networks is available, which uses fiber optic leak detection technology to address deep water pipeline leaks for depths greater than $3000 \mathrm{~m}$ or in arctic conditions.

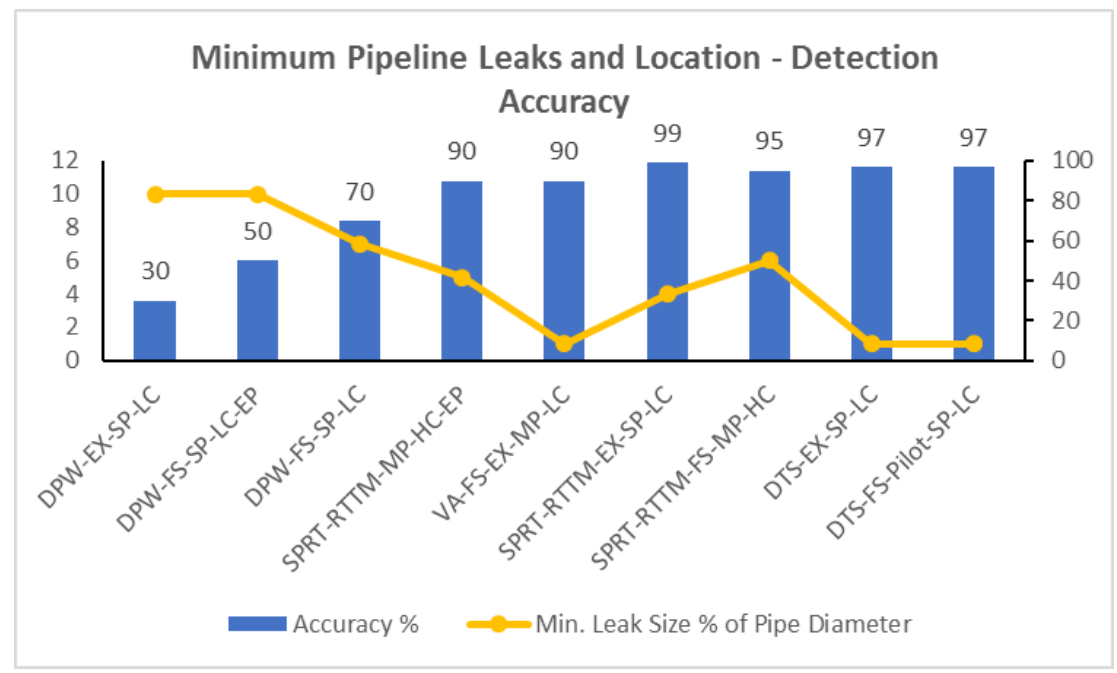

Figure 1 Pipeline Leak and Location Accuracies for Various Leak Sizes 


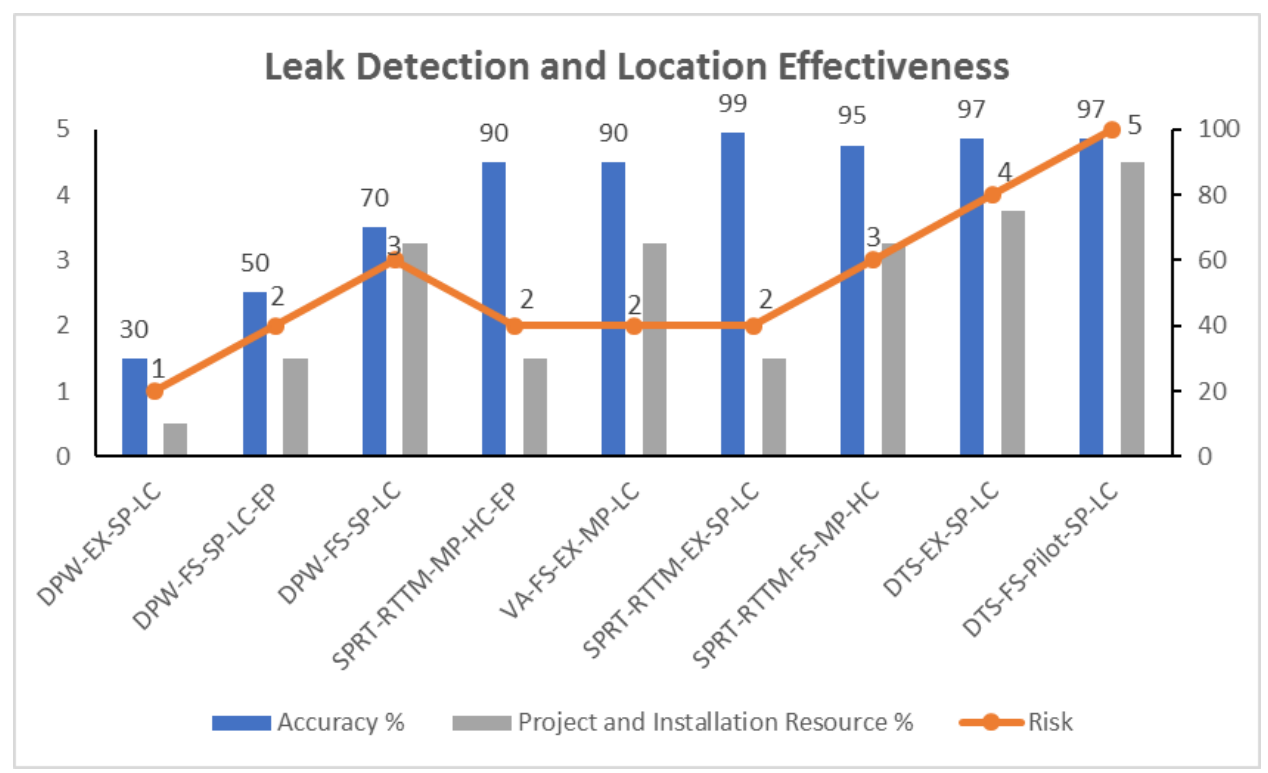

Figure 2 Pipeline Leak and Location Technology Risks and Resource Costs

Table 1 Applications for Various Leak Technologies

\begin{tabular}{|l|l|}
\hline $\begin{array}{l}\text { Leak Detection or Location } \\
\text { Technology }\end{array}$ & Application for Metallic Pipelines \\
\hline DPW-EX-SP-LC & $\begin{array}{l}\text { Onshore Environment with limited applications for liquid } \\
\text { transport in simple pipeline connections e.g. straight runs and } \\
\text { constant operating conditions. The technology is reliant on the } \\
\text { effectiveness of Signal to Noise (SNR) filters and may not be } \\
\text { effective for multiphase flow or for chronic leaks where noise } \\
\text { generated is between 50 to 200Hz }\end{array}$ \\
\hline $\begin{array}{l}\text { SPRT-RTTM-MP-HC-EP } \\
\text { SPRT-RTTM-MP-FS-HC } \\
\text { SPRT-RTTM-EX-SP-LC }\end{array}$ & $\begin{array}{l}\text { Used in new projects due to cost competitiveness and reasonable } \\
\text { accuracy when detecting medium to large leaks greater than }\end{array}$ \\
$\begin{array}{l}\text { 3mm. Can be applied to offshore shallow water conditions and } \\
\text { long range pipelines more than 200km for complex flow } \\
\text { connections }\end{array}$ \\
\hline DTS-EX-SP-LC & $\begin{array}{l}\text { Emerging leak detection and location technology that is not } \\
\text { widely used for arctic and deep water applications. Is more } \\
\text { expensive to install than RTTM and SPRT. Cannot be used for } \\
\text { existing pipeline networks and fiber optic cables can only be } \\
\text { used for fixed lengths to avoid reduction in accuracy }\end{array}$ \\
\hline VA-FS-SP-LC & $\begin{array}{l}\text { Used to monitor small leaks and can be extended to multi phase } \\
\text { flows however it can only be used for small pipes and fixed } \\
\text { lengths }\end{array}$ \\
\hline
\end{tabular}

Figures 1 and 2 with Table 1 show recent technologies used in pipeline leak detection and location together with their accuracies, risk magnitude and resource levels required for installation and operation. Risk magnitude is measured on a scale of 1 to 5 where high installation and operation risk is considered as level 5 and low risk as 1 . The resource level is an approximate percentage based on the fraction of installation and operating cost vs cost of regular pipeline integrity monitoring or where no pipeline leak technology is used. The resource percentage is used as a comparative indicator rather than an absolute measure. The CAPEX for fiber optic leak detection and location is greater compared to existing leak detection systems 
and risk is higher since there are key installation risks related to amplification or repeater units for strengthening the reflected light signal as well as provision for maintenance of the pipeline when the fiber optic cables are installed. Additional installation costs for larger pipe bridges are required due to spacing requirements of the fiber optic cable. Dynamic pressure wave technology is ideal to monitor low hazard pipelines e.g. safe water transport and monitoring of large to medium scaled leaks. SPRT and RTTM has similar accuracy at lower risk compared to fiber optic DTS and is a cheaper alternative to retrofit existing pipeline networks however there is uncertainty for locating or detecting leaks less than $3 \mathrm{~mm}$ for deep water conditions greater than $3000 \mathrm{~m}$ or arctic conditions. Vacuum annulus monitoring uses a pipe-in-pipe arrangement where hydrocarbon leak concentration and location are monitored using high accuracy hydrocarbon metering devices and pressure gauges. The technology can measure leaks smaller than $3 \mathrm{~mm}$ however it cannot be used for large pipeline sizes due to weight of the pipelines, installation difficulties and high CAPEX. During the last decade pipelines for the oil and gas sector manufactured from composite materials or fiber reinforced thermoplastics (RTP) have been successfully used as replacement to metallic pipes due to their light weight and ability to mitigate corrosion effects e.g. hydrogen embrittlement, microbiologically induced corrosion or stress corrosion cracking. The literature reviewed provides no recent field studies for RTP manufactured pipelines using modern leak detection systems for onshore or deep water applications and the effectiveness of leak location or detection could be compromised due to the acoustic damping during pressure wave transmission. Vacuum annulus pressure monitoring using RTP manufactured pipelines can thus be an attractive option for chronic pipeline leak detection.

\section{FIBER OPTIC BASED LEAK DETECTION}

The use of fiber optic (FO) cables is an attractive option for measuring pipeline leak detection and is based on the principle of scattered light that is characterized as Rayleigh, Brillouin, and Raman spectral forms. The Brillouin phenomena occurs when light is scattered due to variations in the refractive index resulting from pressure, temperature or strain. A vibration is produced in the cable from the scattered light that travels at acoustic speed. A frequency downshift on the scattered light results whenever energy is transferred from light photons to the fiber material or a frequency upshift is experienced when photons receive energy from the fiber material. The frequency shift is known as the Brillouin frequency shift [6]. Raman scattering measures temperature changes only and has lower sensing range and longer measurement time compared to Brillouin. For Raman scattering the vibration induced by temperature fluctuation causes low intensity scattered light and high attenuation thus limiting the leak detection range [7]. The ratio of the upshift and downshift frequencies has an exponential relationship with temperature [4,29]. Rayleigh scattering is used in Distributed Acoustic Sensing (DAS) and based on measuring the light signal that is backscattered from the incident light. The backscattered signal has the same frequency and speed as the incident light but travels in the opposite direction. When a leak occurs the distinguished sound level causes changes in backscatter as a function of time that is measured at a receptor and thus the leak location is predicted. There is a $0.2 \mathrm{~dB} / \mathrm{km}$ loss due to the backscatter resulting from the incoming pressure waves and signal strength is dependent on contact between acoustic wave and FOC [4].

The Brillouin Optical Time Domain Analysis (BOTDA) is widely used in oil and gas industry for pipeline leak detection based on strain and temperature changes compared to Rayleigh or Raman scattering techniques. BOTDA uses a square pulsed continuous laser located at either ends. The power derived from

the continuous signal is transferred to the pulsed signal aimed to increase signal to noise ratio (SNR) and sensing range. The probability of false detection decreased as a function of increased temperature change [6].

The FO technology is suitable for onshore applications however there is limited use in offshore applications. The performance characteristics of temperature-based distributed FO cables includes fiber length cable, optical attenuation, data acquisition time and laser pulse width that shines through the FO cable, and temperature sensitivity range between $0.5^{\circ} \mathrm{C}-3^{\circ} \mathrm{C}$. For subsea $\mathrm{FO}$ installation applications the following 
needs to be considered [8]: abrasion and crushing of FO cables, spacing of FO cables, location of tensioner and roller supports, space requirements for FO cable attachment stations on offshore pipelines, damage by submarine activities and weather considerations for arctic applications, e.g. temperature and hydrostatic forces for deep water applications. FO cable repeaters are required every $40 \mathrm{~m}$ to address attenuation and amplification requirements, however the variance in repeatability of the light signal is undermined as a function of FOC length [9].

Brillouin or Raman light scattering can be deployed for Distributed Temperature Sensing (DTS), however Brillouin scattering is the preferred leak detection option. DTS has potential for offshore or arctic applications and uses two or more fiber optic cables installed along the length of a pipeline and connected to a temperature measurement device [3]. DTS technology is also suited for pipeline erosion monitoring at the arctic seabed since the cold water in the event of a leak would reach the soil surrounding the pipeline [10]. Pipeline temperature measurement range using a pulsed laser is usually 30 to $50 \mathrm{~m}$ and can be extended up to $250 \mathrm{~km}$ using signal amplifiers however it cannot be used for subsea applications. Each optical fiber acts as a thermometer and provides temperature distribution resolution coverage over a $2 \mathrm{~m}$ span. For offshore applications DTS is limited to 25 to $30 \mathrm{~km}$ using Brillouin based monitoring measurement devices. Usually DTS is applied to a $60 \mathrm{~km}$ pipe length using these devices on either end or repeater units are installed to extend pipeline coverage to $100 \mathrm{~m}$. Some of the limiting factors for DTS include inability to detect chronic leaks for small temperature variations that is below the fiber optic temperature resolution sensitivity or seabed soil interaction with pipeline temperature distribution might not be uniform at the pipe inlet and outlet or along the length of the pipe. Thus far no published field study data, repeaters or amplifiers are available for FOC leak detection location technology applicable to deep water or arctic pipeline networks.

A combination of distributed strain sensing (DSS) and DTS can be used for onshore underground leak detection for improved accuracy and removing false alarms. For DSS FOC is bonded on a new pipe before installation however there are risks that the FOC can bend or twist thus preventing it from measuring maximum bending strain [3]. The DSS arrangement measures strain and temperature whereas DTS only measures temperature data. For strain measurement the difference is taken between the DTS and DSS signal amplitude. Alternatively there are two bonded and two free FOCs fitted on a thermoplastic profile. Bonded fibers are used for strain measurements whereas the two free fibers measure temperature fluctuations with single redundancy. There is no prescribed method to bond the optic cable to the pipeline for offshore applications and there is risk that the cable may be damaged during the bonding and rolling process. A bonding tape for onshore FOC applications that used fiber glass reinforced thermoplastic (RTP) and a polyphenylene sulfide matrix was developed [11]. The material is bonded under high temperature conditions and provide good mechanical resistance properties, however more tests and modifications are required to the matrix structure for offshore arctic or deep-water applications.

Distributed acoustic sensing (DAS) uses fiber optic to convey acoustic vibration signatures, which is analyzed for leaks, however DAS cannot detect chronic leaks due to background noise and noise generated by the moving fluid. DAS can only be effective for large leaks where the acoustic signal to noise ratio is high and applicable to unburied or buried seabed pipelines. DAS can be used instead of DTS where the fluid temperature measurement is unimportant and detection of large leaks are required [3]. DTS can be effective for normal steady state operations and cannot be used for chronic leak detection especially during transient conditions or when the there is pipe flow start-up due to temperature changes affecting the fluidpipe and pipe-soil interfaces. Other factors affecting DTS and DAS include leak reaction times, soil conditions, single or multi phase fluid mixture and material selection of FOC cable. Plastic optical fibers have higher breaking strains than glass fiber however there is a 700 fold decrease in optical power loss per meter length of cable and the FOC diameter is 100 times larger when compared to using glass FOC [9].

Chronic leak detection using DTS can be affected by the spatial resolution of temperature detected per meter length of pipe. If a chronic leak results in a temperature per meter length that is smaller than the 
spatial resolution then the leak might not be detected. DTS reaction time of 15 minutes to several hours for chronic leak detection needs to be compared with using regular visual aerial inspection and the need for a faster reaction time. Usually large leaks are detected faster compared to chronic leaks [9].

\subsection{Installation and Positioning of FOC for DTS and DAS Applications}

The FOC consists of a core that carries the signal, which is wrapped around by cladding having a different refraction index thus either allowing light energy to the core or retains energy inside it. The core and cladding are then inserted inside a steel or plastic protective jacket. For DAS assembly the FOC consists of the core, acoustic coupling layer with inner interlock, that is housed inside a metal tube and sheath. The DTS has the core inserted in a gel filled stainless steel tube and each tube is surrounded by steel wires. The arrangement is housed in a plastic tube [4].

Signal process and transfer is accomplished using a fiber optic transceiver e.g. a laser with single mode cabling and communication link. The location of FOCs for DTS application is either installing the FOC on a vertical pipeline (12 o clock) section or installing two bundled FOCs at $10 \mathrm{o} \mathrm{clock}$ and $2 \mathrm{o}$ clock positions for improved accuracy [4]. For DAS the FOC is installed 30 to $50 \mathrm{~cm}$ away from the pipe at 3 o clock and 6 o clock positions or if bundling is preferred then the location is like DTS namely at the top pipe section (10 o clock and 2 o clock positions).

\subsection{System Components and Limitations of FOC}

Leak detection FO sensing technology consist of a FO cable and interrogator with sensing range of approximately $50 \mathrm{~km}$ fitted to a processing-control-display unit arrangement. The processing and control unit with leak detection software and graphical interface provides real time pipe flow performance derived from series interrogator units. The leak detection system is integrated to any supervisory control and data acquisition (SCADA) system. The interrogator units analyses acoustic, thermal or strain output signals along the FOC and the software identifies various leak sources based on pattern recognition and data collection.

Limitations of DTS, DSS and DAS were: location, position of orientation and number of fiber optic cables, optimal location of repeaters or amplifiers, and leak detection thresholds for DTS and DAS applications [9]. The literature reviewed to date does not provide actual maintenance activity descriptions for various system components or related mean time between failures (MTBF) however a system and visual maintenance check was recommended every 5 years for fan or automated optical attenuators and 10 years replacement of any fan and system components including hard drive and computers [4]

There were key risks identified for FO sensing technology applied to arctic leak detection namely [4,9]:

- No actual or projected MTBF for individual components or overall leak detection system. MTBF reduces as a function of FO cable length however no optimal MTBF has been determined for various pipeline networks in the arctic or for offshore applications

- Installation and maintenance operational requirements including repeaters used for FO signal transfer in various pipeline sections are not defined

- There are no interrogator or signal amplifying devices available for arctic or subsea applications

- DTS and DAS are unable to quantify chronic leaks or leak rates and there is no established minimum leak threshold

Other competing arctic subsea pipeline leak detection systems for multi phase oil and gas flows are Sequential Probability Ratio Testing (SPRT) used in combination with RTTM, negative pressure wave or 
pressure gradient techniques. Usually SPRT detects leaks whereas negative pressure wave or pressure gradient methods can locate leaks for steady and transient conditions [12].

\section{NEGATIVE OR DYNAMIC PRESSURE WAVES}

A new dynamic pressure wave propagation model and an improved wavelet transform capability model aimed to increase the accuracy of leak detection for natural gas transport in pipelines while excluding time difference effects was developed [13]. The leak location errors for each method were $0.73 \%$ when compared to experimental data. The leak location $\mathrm{x}$, with improvised wavelet transform was given by

$|x|=\frac{\ln \left(P_{2} / P_{1}\right)+\alpha^{+} L}{\alpha^{+}+\alpha^{-}}$

Where

$\mathrm{P}_{1}$ and $\mathrm{P}_{2}$ are the signal amplitude of the upstream and downstream pressures sensors [kPa], $\mathrm{L}$ is pipe span length and alpha are the attenuation factors

And pressure wave is given as

$P(x)=P_{o} \exp \left(\frac{-\alpha+\xi M}{1 \pm M}|x|\right)$

Where

$\xi=$ Froude Number/2xPipe Diameter and $M$ is the Mach number

Based on the amplitude following the pressure wave propagation the upstream and downstream pressures were given by

$P_{1}=P_{o} \exp \left(-\frac{\alpha+\xi M}{1-M}|x|\right)$

$P_{1}=P_{o} \exp \left(-\frac{\alpha+\xi M}{1+M}(L-|x|)\right)$

Field studies for the improvised wavelet transform consisted of a $20 \mathrm{~km}$ natural gas pipeline stretch with $426 \mathrm{~mm}$ diameter and a ball valve of $10 \mathrm{~mm}$ orifice was used to simulate leaks at $1 \mathrm{MPa}$. Various leak sizes and pressures were used in the field study with the aim of obtaining time differences compared to an earlier wavelet transform model and the improvised model. Limited field test for the pressure wave propagation had a pressure range extending from 1 to $5 \mathrm{MPa}$ with leak orifices of 0.45 to $0.8 \mathrm{~mm}$ Maximum error for the old and new wavelet transform were $15 \%$ and $2 \%$ respectively [13]. The errors for the pressure wave propagation model were due to two factors namely: (A) pipeline bends, T-sections, and valve installations that allowed the wave amplitudes to attenuate faster. (B) Mean value parameters based on the sensors located upstream and downstream of the leak were used in the wavelet and pressure propagation equations

A dynamic pressure transmitter (DPT) designed to detect leaks by extracting a wavelet packet entropy (WPE) of the signals was developed [14]. The DPT received low frequency dynamic pressure signals using data acquisition equipment installed at either ends of the pipe. A leak detection monitoring system uses the time difference observed at two ends with speed of the negative pressure wave to determine the leak location. The DPT consisted of piezoelectric transducer, charge amplifier, signal conditioning module, 
micro control unit and power module. The charge amplifier converted high impedance charge to low impedance voltage for long pipeline transport and the energy generated from the acoustic signal was concentrated in the low frequency band for pipeline leaks. A wavelet decomposition strategy was performed on the WPE signals and the wavelet packet coefficients analyzed for signal information. The ratio of energy per wavelet to total energy and the Shannon entropy were then analyzed. The concentration of entropy is calibrated against the pressure range of investigation to identify and locate pipeline leaks. Field experiments were conducted at PetroChina for a $94 \mathrm{~km}$ long pipeline with pipe diameter of $457 \mathrm{~mm}$ and dynamic pressure transmitters installed at the inlet and outlet pipe sections. Pressure signals were obtained for normal operation, valve or pump adjustments and leak scenarios and the time differences for various field tests were recorded with relative error. A maximum error of $0.13 \%$ was observed for 10 sets of field experiments, however these experiments are not conclusive regarding subsea multiphase mixtures or pipeline leaks located on elevation or declines.

Various pressure based leak detection methods for single phase water or oil applications were described in [15]. The hydraulic leak detection method was found suitable for pipeline networks due to faster response times and was less costly. Leak detection using pressure measurements can be used for steady and unsteady state flow seen from figure including the need for vacuum analysis monitoring for chronic offshore leaks.

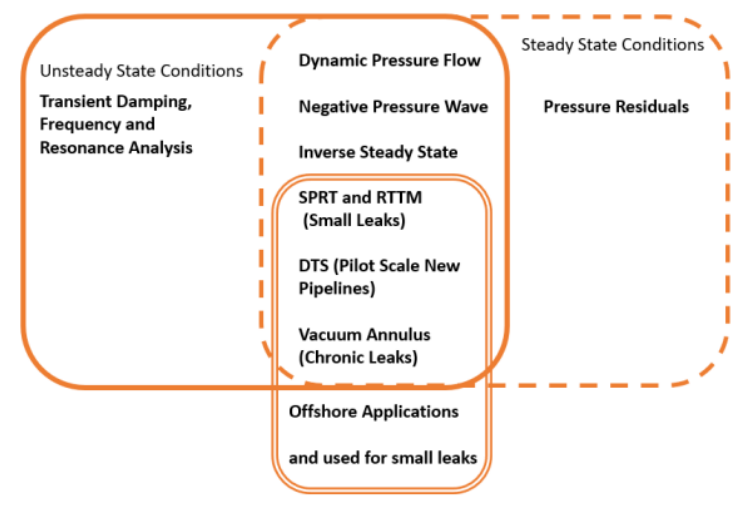

\section{Figure 3 Leak Detection Using Various Pressure Measurement Techniques, Source: Adapted from Abdulshaheed et al, (2017)}

Transient steady state analyses was predicated on properties of the reflected waves and transition waves during a leak. The method uses various pressure and flow data in the pipeline network which was compared with parameters derived from the steady state equations and leak points.

Friction losses were derived using the momentum and energy equations adopting a quasi-static method. The leak location was identified by time differences between the transient and wave reflections, however the technique is applicable to new pipes due to unavailability of deriving friction or roughness data for old or rusted pipes. For unsteady flow the transient damping properties is related to the friction in the pipe and found to be exponential for no leaks and approximately exponential for a leak scenario. Transient waves caused by valve calibration, and pipe joints need to be excluded in the analysis to identify the leak location. A Fourier transform was used on the pressure wave signal and damping rates for leakage conditions and it was found that the ratio of any two damping rates would predict the leak location regardless of leak size. This method can be used to identify chronic leaks for pipelines transporting oil.

The inverse resonance method assumes small pressure transients and the leak discharge is linear with oscillating flow in the pipe network. The leak or valve movement and measured pressure were treated as a transfer function. The flow behavior was solved using either the impedance method or transfer matrix 
method and pressure amplitude oscillations were determined using Fourier decomposition or pseudorandom binary signals (PRBS). The transfer function, pressure oscillations amplitudes and leak locations were located for all frequencies at once using PRBS. Pressure flow deviation works for a wide range of flow conditions and is combined with mass balance leak detection strategy. The leak was located by comparing the measured pressure or flow measurements upstream and downstream of the pipe to predicted pressure and flow variables. The resulting location error and response time were higher for small leaks than large leaks and is not suitable for chronic leak detection. A leak discharge of 5\% total flow produced a 33\% error compared to $22 \%$ error for a $10 \%$ leak discharge. The pressure residual vector was designed for complex pipeline networks transporting water. Pressure and flow sensors installed on the pipeline collect real time data which was compared to a hydraulic simulation model to detect pressure variations or residuals that may cause a leak. Recent pressure simulation models use pattern recognition, regression estimation or neural networks however these models have a 35\% success rate when determining single phase steady state leak detection. Some of the disadvantages of the mass balance approach is accuracy of multiphase flow meters and liquid pipeline hold up [9].

Tian et al [16] developed a statistical process control for transporting oil or gas in pipelines that address challenges related to negative pressure wave leak detection namely, data quality related to missing data, noise level and precision of leak location, data duplication based on time out policy during pressure wave recording and managing false alarms was developed by. Other lessons learnt was frequency filter design can be a trade-off between Signal to Noise Ratio and locating precision requirement. Usually negative pressure wave frequencies derived using pressure transducers measure between 25 to $100 \mathrm{~Hz}$ and communication costs was saved by transmitting data in time packaged batches

Statistical process control (SPC) used a sampling control chart containing various quantitative process measurements, which is compared to controlled sampling distributions. The sampling population is usually pressure data including pressure slope communicated every 10 seconds. Local minimum points were compared to the sampling control chart and updated when required. If pressure data deviated from these local minimum sampling values or control chart then the potential leak time point was calculated. The SPC needs to be used with other leak location technologies e.g. fiber optic, calibrating results from upstream and downstream flow meters, and multiple-sensor pairing algorithm to reduce false alarms or when a pump or valve is adjusted. The sensor pairing recognizes time stamps between sensors and distinguished between pipeline leak or when a pump or valve is actuated, however this technique cannot be used for multi-phase flows or subsea applications 


\section{MAXIMIZING SIGNAL TO NOISE RATIO}

Empirical mode decomposition (EMD) was used to increase SNR based on decomposing various frequency ranges using intrinsic mode functions (IMF) and a residual predicated on a scaled frequency [17]. The IMF has nonlinear characteristics and decomposes the dynamic pressure wave leak signal using cubic splines to identify upper, lower and mean signals. The difference between the source signal and mean signal is inspected for IMF conditions and compared against a predefined acceptable error limit. Decomposition continues until the extreme IMF conditions is less than the acceptable error. The pressure wave amplitudes after denoising from EMD produced the same results as wavelet transform, which should not be confused with Fourier transform.

Another SNR empirical mode decomposition (SNR-EMD) method was developed that consisted of a noise reduction algorithm and smoothing factor which terminated when the noise amplitude factor approached zero [18]. The SNR-EMD technique was used in the field at SINOPEC on a 30k long pipeline for pressure ranges between 4 and $4.15 \mathrm{MPa}$ for crude oil transport. Advantages of this method include removing end effects of noise emanating from the pressure signals

Lately a real time correlation analysis on negative pressure wave for pipeline leak detection transporting flammable gas was devised by Lu et al, [19]. The correlation analysis changes when there is a pressure drop due to a leak and remains constant for steady state operation. The correlation estimation model is represented by

$R_{x y}(\tau)=\frac{1}{T} \int_{-T}^{T} \alpha s(t) s(t-\tau) d t$

Where $s(t)$ is the leakage signal assuming a stationary process, $\alpha$ is a fading factor, $\tau$ is delay time. Environmental noise and leakage signal were assumed irrelevant. A Fourier transform of the upstream and downstream signals $x(n), y(n)$ was done to get $x(k), y(k)$ and the transfer function yielded the mutual power spectral density. The cross correlation function was derived from the Fourier inverse transformation as described in Figure below

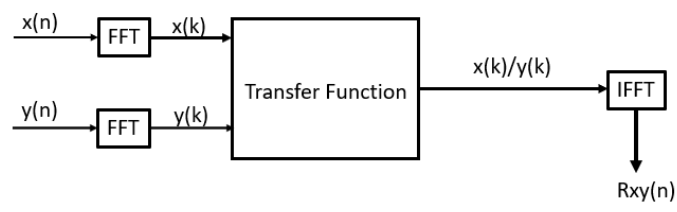

Figure 4 Cross Correlation Function Pressure Drop Source: Adapted from Wang et al, (2018)

Results were simulated using upstream and downstream pressure signals from 20 to $35 \mathrm{kPa}$, including white noise. The wavelet technique was used for denoising and cross correlation analysis preserved low frequency signal characteristics. The cross correlation was used to test for experimental leak detection versus normal operation. 
Adnan et al [20] provided various signal processing methodologies used in pipeline leak detection namely Fast Fourier Transforms (FFT), Short time Fourier Transform (STFT), Wavelet Transforms (WT) and Hilbert Huang Transforms (HHT). The wavelet transform was suited for leaks in gas pipeline transport and uses echoes reflected from turbulence caused by the leak. The echoes are analyzed in the WT algorithm, which reduces the noise using a time based cross correlation technique similar to Wang et al [19]. The HHT technique also designed for gas leak detection in pipelines use acoustic waves from two sensors to obtain the Hilbert marginal spectrum, which compares normal operations and leak conditions. Studies showed that HHT provided more accurate and reliable high resolution time based results compared to FFT and STFT. Spectrograms derived from STFT could clearly indicated when wave amplitudes began to occur during leaks but failed to provide any frequency amplitudes in contrast the WT provided good time and frequency amplitude approximation during leak detection.

The modeling of subsea chronic and large leaks for multi-phase flows in a horizontal pipe by Gajbhiye et al, [21] provides insight to the following:

- $\quad$ Leak positioned further upstream is easier to detect when inlet flowrate and outlet pressure are fixed

- $\quad$ Mixtures that have a compressible gas phase improves leak detection capability due to higher mobility

- $\quad$ The outlet flow rate is a better indicator of leak detection compared to using inlet pressure as a boundary condition

\section{Transient Pressure Wave Leak Detection}

Transient leak detection was addressed by Zhang et [22] for pipelines transporting crude oil since they found pressure transmitters installed along pipelines work well for steady state conditions however there is an increased number of false alarms and leak detection failure due to pumps switching on or off, control valve adjustment and supply changes. A statistical pipeline leak detection (SPLD) system using modified volume balance, pressure and flow monitoring with statistical analysis were combined with a data validation procedure and decision-making methodology. Probability calculations and hypothesis testing for leak or no leak scenarios were based on determining the mass entering or leaving a network and balanced against inventory variations inside a pipeline network. The deviation of the mass balance is detected using a statistical sequential probability ratio test (SPRT) and pattern recognition to avoid spurious or false alarms.

$\tau(t)=\sum_{1}^{M} Q_{1}(t)-\sum_{1}^{N} Q_{o}(t)-\sum_{1}^{L} \Delta Q_{j}(t)$

$\lambda(t)=\lambda(t-1)+\frac{\Delta m}{\sigma^{2}}\left(\tau(t)-m-\frac{\Delta m}{2}\right)$

$\tau(\mathrm{t})$ is the corrected flow imbalance measured at time $\mathrm{t}$, which fluctuates around a non-zero value and $\lambda(\mathrm{t})$ is a cumulative sum based on a leak, no leak hypothesis test with $\mathrm{m}$ as mean value of $\tau(\mathrm{t})$ and $\Delta \mathrm{m}$ is a factor dependent on leak size. The $\sigma 2$ value of relies on pressure and flow fluctuation and the three operating modes namely: steady state operations, small operational change or large operational changes. The SPLD was capable of self learning and adjusted for operational changes or instrument drift in addition to not relying on fluid properties, viscosity change or composition. Field tests for oil transport were analyzed for leaks in a crude oil pipeline network spanning over $173 \mathrm{~km}$. The SPLD identified 47 leaks in a one year period for transient and steady state conditions.

SPRT was used with two statistical volume balance techniques namely Time of Flight (TOF) and pressure interpolation methods using a Least Squares algorithm to predict leak locations [5]. SPRT using the two leak location techniques were applied to 600 pipelines transporting e.g. various crude oil densities, multi 
products: gasoline or diesel, natural gas, ethylene, condensate or diluent. Field tests were conducted extending from Koln to Hamburg Germany for various pipelines elevations.

Real Time Transient Monitoring (RTTM) was replaced with statistical volume balance method since it could detect leaks up to $0.5 \%$ even during transient conditions and not give false alarms during: pump switchover, crude oil changes, start up or shut down, temperature changes, or delivery switchover [5]. The unique ability of statistical volume balance is that leaks of $0.5 \%$ was detected albeit the detection of the flowmeter may have accuracy of $1 \%$.

There are several types of RTTM software applications provided by various suppliers, which may differ in their selection of boundary conditions, or some may include all transients and others would ignore the thermal pipeline effects. Some of the performance measures for RTTM includes [23]:

- Spacing and installation of fluid property measurements (viscosity, mixture, $\mathrm{pH}$ ), and pressure and flow meters such that accuracy of predicted variables are maximized and allows for isolation of the pipeline into independent mass or volume balanced sections

- $\quad$ Size and variability of flows including flows that are exiting the pipeline network

- Managing phase changes especially during pipeline elevations. For instance at low downstream pressures the pressure at the peak may drop to vapor pressure or two phase flow may develop downstream of peak pressure. Thus the correct type of temperature, flow and pressure metering devices need to be installed

- Suitability and selection of boundary conditions and ability to maximize signal to noise ratio

- Detection of a leak with RTTM while compensating for uncertainties and limitations in input data and choice of numerical scheme deployed

RTTM was deployed for a pipeline network that had elevation of $3000 \mathrm{~m}$ above and another section of $2100 \mathrm{~m}$ below sea level [5]. It is difficult to use a RTTM technique to manage pipeline elevations while also accommodating various types of crude oil and a preference was made to the volume balance method.

\section{SUBSEA NEGATIVE PRESSURE WAVE WITH STATISTICAL METHODS}

Leak detection for subsea arctic conditions was addressed by using a combination of sequential probability ratio testing (SPRT), negative pressure wave and pressure gradient signals with pattern recognition integrated in a software algorithm [17]. Wald's SPRT with mass balance and hypothesis testing was used, which assumed the number of samples for signal processing are independent and identically distributed [24]. For subsea multiphase flow transient effects, instrument noise and line packing are major contributors compromising leak detection accuracy thus Wald's SPRT was integrated with a moving-average filtering methodology with normalized data.

WALDS SPRT provides real time leak sample probabilities using moving average filtering to eliminate noise e.g. multiphase transient effects, line packing and instrument air. A hypotheses test is made against a leak or no leak scenario using mean leak probabilities. The difference in mean leak probabilities is identified for no leak scenario and a leak scenario, which is compared to a confidence level of $99 \%$. A larger leak size implies a larger difference in mean leak probability and thus does not meet the required confidence level. Pipeline instrument changes are catered for by the variance and depends on steady state operations, and large to medium changes. Zhang et al, [5, 25] developed SPRT with (RTTM) to predict leak detection using statistical volume balancing for leak location. The technique was successfully applied world wide to 800 pipelines with different dimensions transporting oil or gas at transient and steady state conditions. A highly stable implicit finite difference scheme was used for solving the pipeline equations by developing an adaptive time step and spatial scheme with adjustable accuracy. Field tests were conducted for pipelines 
extending from $2 \mathrm{~km}$ to $2100 \mathrm{~km}$ with diameters between $13 \mathrm{~mm}$ to $2 \mathrm{~m}$ in Sakhalin Russia, Gulf of Mexico, Philippines and Norway. The RTTM system was able to detect leaks ranging from $1 \%$ to $2 \%$ for long distance oil and gas pipelines within 2 hours while $1 \%$ multiphase leaks were detected within 20 hours. Earlier studies showed the SPRT RTTM is a self learning software and continually monitors the relationship between flow and line pressure based on a pattern recognition scheme [26]. Any change in the pipeline pressure and flow relationship would trigger a leak event.

The pressure gradient technique was researched by several authors [23, 27, 22]. For a leak free scenario the pressure drop across a pipeline is a linear real line however during leak conditions the pressure profile develops two gradients and thus forms a kink at the leak point. The location of the leak is at the intersection point of the change in gradients given by the upstream and downstream pressures across the leak. The pressure gradients are calculated using real-time transient monitoring and is suited to multiphase flows.

Negative pressure wave leak detection is based on monitoring the upstream and downstream pressure waves while filtering out noise using multiple pressure sensors at the pipe inlet and outlet. The method is suited for liquid pipeline transport or for high oil to gas ratio applications. Pressure drop during normal operations vs leak conditions or operational changes show different wave forms that need to be evaluated against a predefined or acceptable wave fluctuation. Some of the success factors for effective leak location include: signal processing and wave travel speed and deriving a critical value for pressure drop fluctuations. Simulation studies were compared to field tests for pressure inlet values of 50 bar for a $1 \mathrm{~km}$ pipeline, followed $120 \mathrm{bar}$ for a $10 \mathrm{~km}$ pipeline and maximum errors were ranged between 29 to $4.4 \%$. with maximum error occurring at 20bar. Some of the shortcomings using WALDS SPRT or the pressure gradient techniques include: no provision for quantifying the medium to large operational changes and how these differ from normal operations.

\section{Subsea Vapor Monitoring Chronic Leak Detection}

Bryce et al, [28] developed a hydrocarbon vapor sensing system to identify chronic crude oil leaks in the Arctic called LEOS, which is a German acronym for "Leck Erkennungs Ortungs System" or leak detection and location system. LEOS leak detection was used since pressure variation in a subsea arctic environment can cause hydraulic noise during oil pumping and upstream operations, which can mask chronic leaks.

The LEOS design consisted of a protective braid that covered an outer ethylene vinyl acetate (EVA) sheath perforated PVC tube. A cross woven braid was rolled over a small tube, which contained a gas transport tube, and the pipe assembly was housed in the larger PVC tube. The EVA and tubes allowed C1-C6 hydrocarbon molecules to seep through in the event of a leak. Air and later hydrogen were introduced in the gas test tube for each pump cycle, which created a hydrogen test peak for calibration requirements. Each LEOS detection system was $15 \mathrm{~km}$ long depending on hydrogen sampling concentration and cycle time requirements. Semiconductor gas sensors were used at the measuring station and the logarithmic change in resistivity would indicate the presence of hydrocarbon when compared to the hydrogen test peak. Field tests on the Northstar crude oil pipeline in Alaska was made, which considered subsea pipe-soil interaction, ice gouging and bending strain. Some of the limitations of LEOS is that is can be used for shallow water applications of $15 \mathrm{~m}$ depth and for a maximum pipeline length of $25 \mathrm{~km}$ from [9].

Offshore arctic chronic leaks and large leaks were investigated at 3 sites, namely BP North Star, Pioneer Oooguruk and ENI Petroleum Nikaitchuq [3, 29]. The site at BP utilized primary leak detection using mass balance line compensation that can be measured up to $0.15 \%$ maximum pipe flow and secondary pressure point analysis leak detection for larger leaks applied to oil pipeline transport networks. For chronic leak detection below $0.15 \%$ of maximum pipe flow the LEOS leak detection was used on a daily basis and any hydrogen leak could be measured at various sacrificial anodes [28]. Oooguruk and Nikaitchuq have a threephase mixture of oil, gas and water pipeline network and an arctic heating fuel pipe line. A pipe located 
inside another pipe is used for the three-phase pipeline network and primary leak detection method was the vacuum in the annulus arising from the pipe-in-pipe assembly. Usually the annulus can also be filled with argon at $1 \mathrm{mBar}$ (abs) which improves thermal insulation performance and reduces buckling and frost settlement [29].

Oooguruk and Nikaitchuq had a 12"x16", 6 mile long and 14"x18" at 3.5mile long offshore pipe arrangement respectively. Any pressure increase inside the annulus would indicate a leak regardless of leak hole size and secondary leak detection was comparing the difference of a preset operating pressure safety low with a pressure reduction measurement in the pipe to identify large leaks. A shut in pressure test was conducted to identify leaks in the heating fuel pipe line. A fiber optic sensor cable was installed across the pipeline network and any change in temperature was identified using a distributed sensing algorithm, which prevented false alarms when compared to the primary leak detection deployed. Some of the lessons learnt for subsea arctic leak detection for vapor monitoring incl [3]:

- Chronic leak detection below $1 \%$ of total flow rate and tracking large leaks over a period of 7 days proved effective compared to detecting large leaks within an hour while excluding chronic leak detection

- Technology used to locate chronic leaks should be set at a minimum leak detection threshold

- The minimum leak detection threshold should be compared to the benefits derived vs cost of manufacture, installation, operation and maintenance. The $1 \%$ minimum leak detection threshold may not be suited for all projects

- Pipe-in-pipe arrangement can be costly and incur project delays [4]. Heavy pipe-in-pipe assemblies may require complex or large scale installation machinery and may not be able to be installed or maximum length may be restricted to around 10 miles, however light weight fiber composite pipes with rated pressure may be considered for deep water applications

- Pressure safety low leak monitoring is an imprecise leak detection option since during 1990 and 2001 only 5 out of 21 leaks were positively identified in the Gulf of Mexico using this technique [9]

- Number of sensors, connectors and cabling can be minimized if the monitoring of the annulus is done from one end of the pipeline

- Water tight isolation bulkheads installed on the pipe-in-pipe arrangement are used to isolate sections of the pipe in the event of damaged or water flooding. The bulk heads provide continuous leak detection monitoring however they limit the monitoring capability over the pipeline length

Kulkarni et al, [8] suggested criteria for offshore leak detection, namely: pipeline leak detection coverage of $300 \mathrm{~km}$, spatial leak resolution of 5 to $10 \mathrm{~m}$, provide reliability of $95 \%$ confidence level, able to address chronic leaks, identify leaks within few minute response time, provide leak detection combination of any mixtures containing water, crude oil and natural gas, offshore leak detection equipment should not impact pipeline structures and operate maintenance free. The vapor monitoring system was able to address most of these criteria for onshore applications except the response time needed when a leak was identified due to the sensitivity of measuring the hydrocarbon concentration per cycle time. 


\section{CFD MODELLING SUPPORTING LEAK DETECTION TECHNOLOGY}

Oyedeko et al, [30] provides an elementary description for modelling oil pipeline transport during transient conditions by deriving a one dimensional pipe flow equation, which considers mass flow rate at constant crude oil density. The momentum equation was derived with friction factors for new pipelines and constant pressure drop. The leak location was determined by a discrete processing iterative technique that uses difference in expectation values derived from the mass flowrates of a leak and no leak scenarios. If the discrete processing converges after several iterations then the leak location is positively identified or moves to the next time step. The assumptions used in the model are oversimplified for instance it is restricted to straight horizontal pipe runs for constant diameters applied to pump start-up or shut down. The effects of phase change, complex pipe networks, valve adjustments or pipe leaks at elevations are not addressed and neither is heat losses to the surroundings. Field studies are not conducted and compared to the model to test for robustness and sensitivity. De Sousa et al, [31] expanded on the work of Oyedeko et al, [30] by addressing steady state dynamic behavior of leaking pipelines transporting either oil or water since most CFD studies focused on mass balance using the continuity equation and acoustic behavior caused by the pressure drop across the pipeline to identify location of large leaks. Chronic leaks with leak sizes less than $3 \mathrm{~mm}$ cannot be easily detected using existing mass momentum and pressure based monitoring techniques. These findings are similar to Zhang et al, [5] where a volume based leak rate is preferred over mass and RTTM self learning tool is used instead.

Jujuly et al, [32] provided CFD modelling for pipeline leaks supporting arctic offshore environment using LES turbulent flow to manage high energy eddies. The flow inside the pipe was modelled for steady state transient conditions whereas the leak was modelled for transient conditions only. Both [32,33] found a direct relationship of leak flowrate and pressure drop for incompressible liquids whereas an inverse relationship was observed for ideal gases. Acoustic signatures for leak sizes of 8 and $4 \mathrm{~mm}$ at pressures of 5800 and 200psi respectively were monitored and noise generation was recorded for power spectral density range of 200 to $680 \mathrm{~Hz}[32,34]$. The large eddies create noise disturbances and thus chronic leaks may go undetected for leak diameters less than $3 \mathrm{~mm}$. The mesh selection used in CFD analysis to model pipeline leaks does not provide substantial accuracy with greater mesh refinement and leaks closer to the pipeline entrance provided the greatest pressure drop [35,31]. A medium mesh element range of 210,000 to 600,000 is adequate for pipeline leak detection.

Subsea oil and gas pipeline leaks including free surface effects showed that high density oil and small leak diameter with minimum flow rates created maximum horizontal migrate distances for an oil sheen appearing on the water surface [36,37]. For chronic leaks at minimum gas flow rates the turbulence at the water surface is less pronounced however there is no CFD research available to adequately predict deep water or arctic gas leak plumes at the water surface. The reaction times for the oil sheen or gas mixture formation on the water surface at steady state conditions from initiation of a subsea leak needs to be fully understood and compared to the reaction times of modern leak detection systems.

\section{RESEARCH GAPS IDENTIFIED IN LEAK DETECTION SURVEY}

Fiber optic cable used in leak detection is an emerging technology for deep water and arctic conditions that is confined to pilot scaled studies. Some of the installation shortcomings include: attachment of cable on the pipe from a tensioned roller for deep water applications, location of the cable on the pipe bridge and spacing constraints, provision of maintenance activities on pipeline networks e.g. welding and removal of pipe sections, retrofitting of existing pipelines with FOC and space restrictions on pipe bridges. There are no amplifiers or repeater signal generators for offshore conditions. Installation and design costs are greater than other leak detection technologies due to installation risks and equipment reliability is not fully 
established. For DSS applications the sealing of the cable on pipe for offshore conditions need to be investigated using the RTP sealing technique on metallic or RTP pipes.

Dynamic pressure wave leak detection can be used with a customized SNR filter however the Fourier transform algorithm may not be ideally suited for transient conditions, multiphase flows or when there is a flow change, pipe elevation, pump start-up or shutdown. Further research is required for RTTM with SPRT and dynamic pressure wave to address chronic leaks for deep water offshore environment. Chronic leak signals are generated for frequency less than $200 \mathrm{~Hz}$ and further analysis is required to identify calibration of pressure signals, frequency generated and leak sizes between 1 to $3 \mathrm{~mm}$. The challenge is to identify the noise generated especially for multiphase flows and the signal generated in the event of chronic leaks for the frequency range between 30 to $200 \mathrm{~Hz}$.

Vapor Monitoring Chronic Leak Detection has proven to be effective for chronic leak detection however further improvement is required to lower the weight of the pipe in pipe configuration for easy assembly and installation in arctic or subsea conditions. RTP is an attractive alternative to metallic pipeline networks however there is uncertainty regarding fracture effects of the pipes in harsh conditions or external interference. Further investigation is required to compare the effectiveness of existing leak detection systems using RTP pipeline networks.

Shortcomings in CFD Modelling include the flow dispersion during transient and steady state as a function of time for deep water and arctic conditions. The time for full pool formation or oil sheen needs to be characterized for the various leak detection reaction times. It is unclear how oil or gas dispersion would be affected in deep water or during ice build up around the submerged pipe. Field studies for leak dispersion or oil sheen formation is required and compared to the accuracy and reaction for various leak sizes and leak technologies since no one size fits all leak detection application may be able to address chronic or large leaks.

\section{SUMMARY DISCUSSION ON LEAK DETECTION SURVEY}

FOC provides higher accuracy and temperature vs frequency resolution compared to other leak detection technologies at maximum accuracy. Both DTS and DAS require minimal maintenance, however the installation costs on new pipelines is greater than existing leak detection location technologies like negative pressure wave or SPRT with RTTM. No technology is available to retrofit existing oil and gas pipelines and neither is this technology used for deep water or arctic applications. The sealing of FOC on pipelines and signal amplifiers or repeaters are currently unavailable for subsea arctic applications. Chronic leak detection and location for subsea oil and multiphase gas mixtures are best suited using either RTTM and SPRT or pipe-in-pipe vapor monitoring configuration using fiber composite material due to the light weight composition, less cost and easy to install process especially for large diameter pipelines exceeding $400 \mathrm{~mm}$ diameter. For FOC to be successfully deployed for offshore applications a self learning or artificially intelligent system with RTTM maybe useful to address transient conditions.

Igor et al, [38] provides a useful description about how fiber composite manufactured pipelines can be a game changer for oil and gas transport and key findings include moisture absorption and subsea pipeline repair strategy given in [39-41], A description in [42] for installation of buried fiber composite pipelines and guidelines on pipe-in-pipe installations. Oil and gas pipeline flow networks utilizing Reinforced Thermoplastic Pipes (RTP) has been in operation at Saudi Aramco to mitigate corrosion effects related to $\mathrm{CO} 2, \mathrm{H} 2 \mathrm{~S}, \mathrm{O} 2$ and bacterial growth [45]. RTP manufactured subsea umbilicals or pipelines can be customized for deep sea applications and their strength and bending characteristics is described by $[43,44]$. RTP manufactured risks include fracture or crack propagation of the pipe in the event of external interference e.g. sand deposition in the pipe network, earth disturbances or ice formation around a submerged pipe. 
Negative or dynamic pressure wave technology can be applied for large scaled leaks typically for leaks that are $10 \%$ of maximum flow rate or greater due to location errors and sub optimal signal to noise ratio filtering. The signal to noise ratio (SNR) methods adopted are like other SNR filtering techniques available from various pipeline leak detection suppliers $[17,19]$. Some of the challenges deriving maximum SNR is transient conditions in pipeline networks e.g granular SNR filtering during valve motion, pump start-up, addressing multiphase flows and pipeline elevations. The transient modes described are fully catered when using RTTM and the calibration table indicates more consistent and minimal leak detection errors using SPRT and RTTM when compared to negative pressure wave leak location even when using sophisticated SNR software. In addition no large scale field tests describing leak location or leak detection of complex pipeline network systems is available in the literature utilizing negative or dynamic pressure wave technology.

Transient pressure wave leak detection using SPRT with RTTM proves to be the best leak detection and location technique for existing complex pipeline networks transporting single or multiphase mixtures and can be used even on pipeline elevations and offshore. Installation is easy and the cost is similar to the negative pressure wave leak detection system. There is no self learning tool in other leak detection systems to address transient effects in pipeline pressure, flow or temperature. The RTTM allows for self correction in the event of mixture change, pressure, flow or temperature transients. Even chronic leaks can be easily identified and located with greater accuracy compared to flow or pressure instruments. Recent developments indicate that the volume balance method is preferred over mass balance for greater accuracy especially during pipeline elevation when transporting multiphase flows in subsea or arctic conditions. Usually pressure or flow transmitters are unable to measure either liquid, gas or a mixture within reasonable accuracy especially for pipeline networks at steep elevations to the vertical or during phase change of mixture composition for arctic or deep water applications. RTTM and SPRT is the preferred leak detection location technology used in Royal Dutch Shell [46].

Existing pipeline leak CFD analysis focused on above ground or shallow water single phase flows for single mode transient conditions e.g. pump start-up or shut down while using an outdated mass balance technique to determine leak rates and the momentum transfer equation. The disadvantages of this approach is that multiphase flows cannot be easily predicted especially for pipeline leaks at elevations since the flow becomes stratified and the liquid-vapor mixture may flash depending on the operating pipeline pressure. Moreover leak detection and location systems do not have reaction times from the initiation of a leak till there is steady state leak rate and dispersion formation. The steady state formation of oil sheens on the water surface for deep water pipeline networks and arctic conditions have not been sufficiently investigated when compared to the reaction times of modern leak detection systems.

\section{CONCLUSION AND RECOMMENDATIONS}

Popular leak detection techniques that are validated with field studies include: RTTM and SPRT, negative or dynamic pressure wave utilizing intelligent SNR technology. There is limited use of FOC leak detection system especially for deep water or arctic applications. The highest accuracy and most cost effective leak detection and location systems is RTTM with SPRT that has self learning capability, which can be retrofitted on existing pipelines and even for multiphase flows at elevations and offshore conditions. Negative or dynamic pressure wave is another attractive low cost leak location option for existing pipelines that provides higher accuracy for increasing leak sizes and may not be suitable for chronic leaks less than $4 \mathrm{~mm}$. These technologies currently do not support lightweight pipelines manufactured from composite 
materials or reinforced thermoplastic application due to the damping effects and loss of signal to noise ratio. FOC using DTS, DAS or DSS is suitable for new above ground pipeline installations due to additional accuracy however there is risk of damage to the optic cable in the event of any subsequent pipeline repair or replacement. There is currently no established complex pipeline installations using FOC for deep water and arctic applications. Some of the recommendations for improving leak detection and location technologies supported by CFD pipeline leak dispersion analysis for arctic and deep water applications include:

- Develop a lightweight easy to install pipe-in-pipe assembly for vacuum based pressure monitoring. The adoption of FOC using DTS or DAS using Brillouin based leak detection can make an ideal leak detection system since large and chronic leaks can be easily detected

- Develop FOC sealing technologies using reinforced thermoplastic to bond FOC on existing pipelines and new fiber composite pipelines. These pipelines are lightweight and safeguard against pipeline corrosion failure modes for hydrocarbon gas and liquid transport

- Understand the behavior of multiphase leak dispersion for deep water and arctic applications for elevated pipelines focusing on chronic leaks and formation of any oil sheen or gas-liquid breakthrough formation at water surface for steady state conditions. The reaction times of leak detection technologies should be compared to the actual leak quantity and oil sheen or gas-mixture formation at the water surface

- Develop new amplifiers or repeaters to strengthen reflected light signals for deep water and arctic conditions since these devices need to be installed above the water surface. Mean time between failures on individual components supporting FOC installations for extreme weather at sea level depths more than $3000 \mathrm{~m}$ are unknown and pilot studies are thus required to derive MTBF failure data and established FOC installation and repair practices 
APPENDIX TABLE A LEGEND FOR LEAK DETECTION TECHNOLOGIES

\begin{tabular}{|l|l|}
\hline Legend & Description \\
\hline DPW & Dynamic Pressure Wave \\
\hline DTS & Distributed Temperature Sensing \\
\hline VA & Vacuum Annulus \\
\hline RTTM with SPRT & Real Time Transient Monitoring with Sequential Probability Ratio Test \\
\hline FS and EX & Field Study or Experiment \\
\hline EP & Existing Pipelines \\
\hline SP and MP & Single Phase or Multiphase \\
\hline LC or HC & Low Complexity or High Complexity Pipeline Connections \\
\hline
\end{tabular}

\section{APPENDIX TABLE B CALIBRATION TABLES FOR LEAK DETECTION TECHNOLOGIES}

\begin{tabular}{|c|c|c|c|}
\hline $\begin{array}{l}\text { Fiber Optic } \\
\text { Model }\end{array}$ & Authors & $\begin{array}{l}\text { Operating Conditions incl. } \\
\text { Accuracy }\end{array}$ & Critical Findings \\
\hline Raman Scattering & [6] & $\begin{array}{l}\text { Sensing range of } 37 \mathrm{~km} \text {, } \\
\text { measurement time }<3 \mathrm{~min} \\
\text { Temperature accuracy of } \\
3^{\circ} \mathrm{C}\end{array}$ & \\
\hline $\begin{array}{l}\text { Brillouin } \\
\text { Scattering }\end{array}$ & {$[29,4]$} & $\begin{array}{l}50 \mathrm{~km} \text { sensing rang, Less } \\
\text { than } 1 \mathrm{~m} \text { spatial resolution, } \\
1 \mathrm{~min} \text { measuring time, } 2^{\circ} \mathrm{C} \\
\text { resolution }\end{array}$ & $\begin{array}{l}\text { Probability of False Alarms decrease } \\
\text { with increase temperature change } \\
\text { Detection occurs when measured } \\
\text { signal power is larger than } \\
\text { predetermined threshold. Large } \\
\text { number of scans increases signal to } \\
\text { noise ratio and probability of detection }\end{array}$ \\
\hline DTS & [3] & $\begin{array}{l}25 \text { to } 30 \mathrm{~km} \text { sensing range } \\
\text { Resolution coverage spans } \\
2 \mathrm{~m}\end{array}$ & $\begin{array}{l}\text { Can use repeaters to extend range but } \\
\text { proves difficult for offshore } \\
\text { applications. Installed above water at } \\
25 \mathrm{~km} \text { intervals }\end{array}$ \\
\hline DTS and DAS & {$[4,29]$} & $\begin{array}{l}\text { Interrogator Units of } \\
1550 \mathrm{~nm} \text { optical wave } \\
\text { length. Sampling rate of } 2 \text { to } \\
2.5 \mathrm{kHz} \text { per } 50 \mathrm{~km} \text { operating } \\
\text { range. Acquisition time of } \\
\text { interrogator between } 1 \mathrm{sec} \text { to } \\
5 \mathrm{~min}\end{array}$ & Leak detection to $1 \%$ of pipe flow rate \\
\hline Raman Scattering & [4] & $\begin{array}{l}\text { Sensing range between } 8 \text { to } \\
10 \mathrm{~km}\end{array}$ & \\
\hline \multirow[t]{2}{*}{$\begin{array}{l}\text { Rayleigh DAS } \\
\text { Scattering }\end{array}$} & [4] & $\begin{array}{l}\text { Sensing range between } 45 \text { to } \\
50 \mathrm{~km} \\
0.5 \mathrm{~dB} / \mathrm{km} \text { loss in } \\
\text { backscattered signal }\end{array}$ & \\
\hline & [29] & & \\
\hline
\end{tabular}




\begin{tabular}{|c|c|c|c|}
\hline $\begin{array}{l}\text { Leak Detection } \\
\text { Experimental Data }\end{array}$ & Authors & $\begin{array}{l}\text { Operating Conditions incl. } \\
\text { Accuracy }\end{array}$ & Critical Findings \\
\hline $\begin{array}{l}\text { Vacuum Annulus } \\
\text { Monitoring }\end{array}$ & [29] & $\begin{array}{l}\text { Pipe-in-Pipe Annulus pressure } \\
1 \mathrm{mBar}(\mathrm{abs}) \text { with Argon Gas }\end{array}$ & \\
\hline $\begin{array}{l}\text { Distributed } \\
\text { Temperature Sensing }\end{array}$ & [9] & $\begin{array}{l}\text { Uninsulated gas pipeline test } \\
\text { length of } 200 \mathrm{~m} \text { with leak rate } \\
\text { of } 1201 / \mathrm{min} \text {. DTS detected a } 2 \\
\text { Deg C change }\end{array}$ & \\
\hline $\begin{array}{l}\text { Dynamic Pressure } \\
\text { Wave }\end{array}$ & [13] & $\begin{array}{l}\text { Experiment Setup } \\
\text { Compressed air at } 5 \mathrm{MPa} \text { and } \\
\text { pipe length under } 200 \mathrm{~m} \text { with } \\
\text { diameter } 10 \mathrm{~mm} \text { having } 3 \\
\text { leakage points used for test } \\
\text { Field Setup (Shengli Oil } \\
\text { Field) } \\
\text { Pipe length of } 20 \mathrm{~km} \text { and } \\
\text { 426mm diameter with } 8,7,6,5 \\
\text { and } 10 \mathrm{~mm} \text { leak orifices. } \\
\text { Operating pressure of } 1 \text { to } \\
5 \mathrm{MPa} \text { at } 300.65 \mathrm{~K} \text { with } \\
15720 \mathrm{~m} 3 / \mathrm{hr} \text { flow rate. } \\
\text { Frequency amplitude between } \\
0-50 \mathrm{~Hz}\end{array}$ & $\begin{array}{l}\text { Leak detection with orifice to pipe } \\
\text { diameter ratio of } 1 \% \text { and error of } \\
0.1 \% \text { for experiments. } \\
\text { Greater time difference accuracy } \\
\text { using modified wavelet transform for } \\
\text { effective leak location } \\
\text { Maximum location errors for } \\
\text { experimental set up } 1.9 \text { to } 2.03 \% \\
\text { upstream and downstream } \\
\text { respectively. } \\
\text { Maximum location errors for Field } \\
\text { set up } 2 \text { to } 9 \% \text { upstream and } \\
\text { downstream respectively. } \\
\text { Unsuitable for multiphase flows or } \\
\text { chronic leaks less than } 3 \mathrm{~mm}\end{array}$ \\
\hline
\end{tabular}




\begin{tabular}{|c|c|c|c|}
\hline $\begin{array}{l}\text { Leak Detection Field } \\
\text { Study Data }\end{array}$ & Authors & $\begin{array}{l}\text { Operating Conditions incl. } \\
\text { Accuracy }\end{array}$ & Critical Findings \\
\hline $\begin{array}{l}\text { SPRT and Volume } \\
\text { Balance }\end{array}$ & {$[25]$} & $\begin{array}{l}\text { Sakhalin } 1 \text { Russia } \\
\text { Pipe length 202km and Diameter } \\
\text { 48" (Crude Oil) } \\
\text { Pipe length 17km Diameter 24" } \\
\text { (Natural Gas) } \\
\text { Sakhalin } 2 \text { Russia } \\
\text { 1600km (onshore) } \\
\text { 300km (offshore) } \\
\text { 13km (multiphase) } \\
\text { Water Depth 50m } \\
\text { (oil, gas, mono-ethylene glycol, } \\
\text { and multiphase combination) } \\
\text { Gulf of Mexico (Crude oil) } \\
\text { Caesar Pipeline Water depth of } \\
\text { 1524m and 185km long } \\
\text { Cameron Highway 612km and } \\
\text { diameter range between } 24 " \text { to } 30 " \\
\text { Philippines } \\
\text { Natural Gas Pipeline 504km } \\
\text { Norway } \\
\text { Natural Gas subsea pipeline } \\
\text { network } 7800 \mathrm{~km} \text { in North Sea } \\
\text { With diameters up to } 1.18 \mathrm{~m}\end{array}$ & $\begin{array}{l}\text { Long oil pipelines leak } \\
\text { detection system (LDS) } \\
\text { detects } 1 \% \text { in } 2 \text { hours and } 5 \% \\
\text { in } 15 \text { minutes } \\
\text { Long gas pipelines LDS } \\
\text { detects } 2 \% \text { in } 2 \text { hours and } 5 \% \\
\text { in } 40 \text { minutes } \\
\text { Multiphase pipelines LDS } \\
\text { detects } 1 \% \text { in } 20 \text { hours and } 5 \% \\
\text { in } 40 \text { minutes } \\
\text { Cameron Highway: } \\
1 \% \text { leak detected in } 1 \text { hour } \\
5 \% \text { leak in } 15 \text { minutes } \\
\text { Pipeline Management System } \\
\text { provides reliability, production } \\
\text { and gas quality data incl. leak } \\
\text { detection }\end{array}$ \\
\hline & {$[5]$} & $\begin{array}{l}\text { Germany } \\
\text { Crude Oil Pipeline } \\
\text { Pipe length from } 2 \mathrm{~km} \text { to } 2100 \mathrm{~km} \\
\text { Pipe Diameter } 0.5 \text { " to } 80 " \\
\text { Elevation from } 2100 \mathrm{~m} \text { below to } \\
3000 \mathrm{~m} \text { above sea level }\end{array}$ & $\begin{array}{l}\text { Replaced RTTM with volume } \\
\text { balance system due to } \\
\text { suppliers inability to identify } \\
\text { leaks during transient } \\
\text { operations and provides } 0.25 \% \\
\text { leak detection }\end{array}$ \\
\hline $\begin{array}{l}\text { SPRT and Volume } \\
\text { Balance Method }\end{array}$ & [5] & $\begin{array}{l}\text { Pressure }=0.3 \mathrm{Bar} \\
\text { Pipeline Length }=173 \mathrm{~km} \\
\text { Temperature }=10 \text { Deg C } \\
\text { Crude oil density between } 798 \text { to } \\
925 \mathrm{~kg} / \mathrm{m}^{3} \\
\text { Viscosity between } 2.8 \text { and } 309 \mathrm{cst}\end{array}$ & $\begin{array}{l}\text { Self learning tool adjusts for } \\
\text { steady state and transient } \\
\text { operations and identified } 47 \\
\text { leaks/year. Robust LDS for } \\
\text { single phase flow }\end{array}$ \\
\hline $\begin{array}{l}\text { Pipe Annulus } \\
\text { Hydrocarbon } \\
\text { Monitoring (LEOS) } \\
\text { and Vacuum } \\
\text { Monitoring }\end{array}$ & {$[3,28]$} & $\begin{array}{l}\text { Mass balance and pressure point } \\
\text { analysis used for larger leaks up to } \\
0.15 \% \text { of mass flow } \\
\text { Offshore Oil Pipeline lengths are } \\
12 \text { "x16", } 6 \text { mile long and } 14 \text { "x18" } \\
\text { at } 3.5 \text { mile long }\end{array}$ & $\begin{array}{l}\text { Used for chronic leaks even } \\
\text { below } 1 \% \text { and subsea } \\
\text { applications at BP North Star, } \\
\text { Pioneer Oooguruk, and ENI } \\
\text { Petroleum Nikaitchuq. } \\
\text { Suitable for chronic onshore } \\
\text { and subsea leaks. Can be used }\end{array}$ \\
\hline
\end{tabular}




\begin{tabular}{|l|l|l|l|}
\hline & & & $\begin{array}{l}\text { for short pipe length due to } \\
\text { size and weight of the pipes }\end{array}$ \\
\hline $\begin{array}{l}\text { Negative or Dynamic } \\
\text { Pressure Wave }\end{array}$ & {$[13]$} & $\begin{array}{l}\text { Natural Gas Pipeline Length }= \\
\text { 20km } \\
\text { Diameter=426mm } \\
\text { Pressure Range of 1 to 5Mpa } \\
\text { using wavelet transformation } \\
\text { and error location of 2\%. } \\
\text { Existing wavelet transforms } \\
\text { addressing SNR is 15\% } \\
\text { Can be used at shallow depth } \\
\text { of less than 2m and unsuitable } \\
\text { for plastic pipes } \\
\text { Cannot be used for chronic } \\
\text { leaks }\end{array}$ \\
\hline $\begin{array}{l}\text { Dynamic Pressure } \\
\text { Transmitter with } \\
\text { Wavelet Packet } \\
\text { Entropy }\end{array}$ & {$[14]$} & $\begin{array}{l}\text { Petrochina } \\
\text { Oil Pipeline length is } 94 \mathrm{~km} \\
\text { Pipe diameter is } 457 \mathrm{~mm}\end{array}$ & $\begin{array}{l}\text { 10 Field tests conducted in } \\
\text { PetroChina with error of } \\
0.13 \% \text { for leak size larger than } \\
10 \mathrm{~mm}\end{array}$ \\
\hline
\end{tabular}

\begin{tabular}{|c|c|c|c|}
\hline $\begin{array}{l}\text { Leak Detection CFD } \\
\text { Modelling }\end{array}$ & Authors & $\begin{array}{l}\text { Operating Conditions incl. } \\
\text { Accuracy }\end{array}$ & Critical Findings \\
\hline \multirow[t]{3}{*}{ CFD Simulation } & {$[31]$} & $\begin{array}{l}\text { Water density }=998.2 \mathrm{~kg} \\
\text { Viscosity }=0.001003 \mathrm{~kg} / \mathrm{ms} \\
\mathrm{V}=0.1 \mathrm{~m} / \mathrm{s} \\
\text { Pipeline length }=1 \mathrm{~m} \\
\text { Diameter }=0.15 \mathrm{~m} \\
\text { Temperature }=25 \text { Deg C } \\
\text { Leak Diameters } 1 \%, 5 \% \text { and } 10 \% \\
\text { pipe diameter }\end{array}$ & $\begin{array}{l}\text { Steady State Leak scenarios } \\
\text { Medium mesh size of } 200,000 \\
\text { to } 600,000 \text { element size is } \\
\text { adequate for leak dispersion }\end{array}$ \\
\hline & [32] & $\begin{array}{l}\text { Pipe length }=8 \mathrm{~m} \text {, } \\
\text { Diameter }=0.32 \mathrm{~m} \\
\text { Mesh elements less than 3million } \\
\text { With 3-D unstructured triangular } \\
\text { mesh } \\
\text { Leak orifice sizes between } 4 \text { to } \\
8 \mathrm{~mm} \\
\text { Pressure range of } 200 \text { to } 5800 \mathrm{Psi} \\
\text { Pipe Temperature Range of } 277 \text { to } \\
320 \mathrm{~K} \\
\text { Ambient Pressure of } 150 \mathrm{psi} \text { at } \\
\text { leak outlet } \\
\text { Sea level depth of } 100 \mathrm{~m}\end{array}$ & $\begin{array}{l}\text { Transient Analysis done for } \\
\text { methane, oil, water and } \\
\text { Nitrogen } \\
\text { Shallow water CFD analysis } \\
\text { for medium to large leaks. } \\
\text { Leak rate is function of } \\
\text { pipeline pressure for liquids } \\
\text { and leak rate is inversely } \\
\text { proportional to pipeline } \\
\text { differential temperature } \\
\text { Power spectral density range } \\
\text { of } 220 \text { to } 500 \mathrm{~Hz}\end{array}$ \\
\hline & [34] & $\begin{array}{l}\text { Pipeline length }=2 \mathrm{~m} \\
\text { Diameter }=0.1 \mathrm{~m} \\
\text { Small rectangular hole of } 1 \times 1 \mathrm{~mm} \\
\text { Water velocity }=1 \mathrm{~m} / \mathrm{s} \\
\text { Pressure Range of } 1 \text { to } 5 \mathrm{bar}\end{array}$ & $\begin{array}{l}\text { Ideal to approximate large } \\
\text { transient single phase leaks } \\
\text { Power spectral density (PSD) } \\
\text { range of less than } 200 \mathrm{~Hz} \text { for } \\
\text { plastic pipes and large sized }\end{array}$ \\
\hline
\end{tabular}




\begin{tabular}{|c|c|c|c|}
\hline & & $\begin{array}{l}\text { Other rectangular hole sizes: } \\
2 \times 2 \mathrm{~mm} \text { and } 10 \times 10 \mathrm{~mm}\end{array}$ & $\begin{array}{l}\text { leaks of } 10 \times 10 \mathrm{~mm} \text { the PSD is } \\
250 \text { to } 400 \mathrm{~Hz}\end{array}$ \\
\hline $\begin{array}{l}\text { CFD Model } \\
\text { Development }\end{array}$ & [30] & $\begin{array}{l}\text { Elementary model using mass and } \\
\text { momentum equations with } \\
\text { discrete leak location time } \\
\text { marching solution } \\
\text { Water density }=834.2 \mathrm{~kg} \\
\text { Viscosity }=0.00172 \mathrm{Pas} \\
\text { Pipeline length }=2 \mathrm{~m} \\
\text { Diameter }=0.36 \mathrm{~m} \\
\text { Inlet Temperature }=295.2 \mathrm{~K} \\
\text { Outlet Temperature }=293.4 \mathrm{~K} \\
\text { Outlet Mass flow rate }=71.48 \mathrm{~kg} / \mathrm{s} \\
\text { Inlet Mass flow rate }=71.45 \mathrm{~kg} / \mathrm{s}\end{array}$ & $\begin{array}{l}\text { Oversimplified assumptions } \\
\text { used and model cannot cater to } \\
\text { various transient modes or } \\
\text { dynamic behavior of a } \\
\text { complex pipeline network } \\
\text { Can be used for rudimentary } \\
\text { approximation of leaks in new } \\
\text { pipelines }\end{array}$ \\
\hline
\end{tabular}




\section{REFERENCES}

[1] CEPA, Canadian Environmental Protection Agency, What Are Pipeline Companies Doing About Climate Change, www.aboutpipelines.com, Date 4th Sept 2019. Book

[2] Behari N., Detection of Hydrocarbon Leaks and its HC Emission Quandary, AWE International Magazine, Issue 57, Buxton Press Limited, UK, January 2019, Book

[3] Eisler B., Leak Detection Systems and Challenges For Arctic Subsea Pipelines, Offshore Technology Conference, Article 22134, Houston Texas, March 2011, Conference Proceedings

[4] Thodi P., Paulin M., Forster L., Burke J., and Lanan G., Arctic Pipeline Leak Detection using Fiber Optic Cable Distributed Sensing Systems, Arctic Technology Conference, OTC 24589, Houston, February 2014, Conference Proceedings

[5] Zhang J., Hoffman A., Kane A., and Lewis J., Development of Pipeline Leak Detection Technologies, 10th International Pipeline Conference, Calgary Alberta, Canada, 2014, Conference Proceedings

[6] Aljaroudi A., Khan F., Akinturk A., Haddara M., and Thodi P., Probability of Detection and False Alarm for Subsea Leak Detection Systems: Model and Analysis, Journal of Failure Analysis and Prevention, Vol 15 pp 873-882, 2015, Journal

[7] Geiger G., State of the Art Leak Detection and Localization, Pipeline Technology Conference, 2006, Conference Proceedings

[8] Kulkarni M., Buitrago J. and Arslan H., Offshore Pipeline Leak Detection System Concepts and Feasibility Study, Proceedings of the Twenty-second International Offshore and Polar Engineering Conference, Rhodes, Greece, June 2012, Conference Proceedings

[9] Eisler B., Lanan G., Lanan G.A., Fiber Optic Leak Detection Systems For Subsea Pipelines, Offshore Technology Conference, Article 23070, Houston Texas, May 2012, Conference Proceedings

[10] Eisler B., Fiber Optic Temperature Sensing Systems Monitor Subsea Pipelines for Erosion and Leaks, Materials Performance, 47-8, ProQuest Materials Performance, August 2008, Conference Proceedings

[11] Inaudi D. and Glisic B., Long-Range Pipeline Monitoring by Distributed Fiber Optic Sensing, Journal of Pressure Vessel Technology, ASME Volume 32, February 2010, Journal

[12] Bai Y., Zhang T., Li Y., Ruan W., and Zhu K., A new leak detection method for subsea pipelines, Ships and Offshore Structures, Vol 12:sup, S144-S152, 2017, Journal

[13] Liu C., Wang Y., Li Y. and Xu M., Experimental Study on new leak location methods for natural gas pipelines based on dynamic pressure waves, Journal of Natural Gas Science and Engineering, Vol 54, 83-91, March 2018, Journal

[14] Zhang Yu, Chen S. and Jin S., Leak Detection Monitoring System of long distance oil pipeline based on dynamic pressure transmitter, Journal of Measurement, Vol 49, 382-389, 2013, Journal

[15] Abdulshaheed A., Mustapha F., and Ghavamian A., A pressure based method for monitoring leaks in a pipe distribution, Renewable and Sustainable Energy Reviews, Vol 69, 902-911, 2017, Journal

[16] Tian H. Yan C.J., Huang J., Wang Yu, Kim D.S. and Yi Tongnyoul Yi, Negative Pressure Wave based Pipeline Leak Detection: Challenges and Algorithms, IEEE International Conference on Service Operations and Logistics, and Informatics, pp 372-376, July 2012, Conference Proceedings

[17] Liu Wei, Liu Hongzhao, Research on Automatic Identification for the Leakage Signal of Petroleum Pipeline, Sensor and Transducers, Vol 21, Special Issue, pp 147-152, May 2013, Journal 
[18] Lu W., Liang W., Zhang L. and Liu W., A novel noise reduction method applied in negative pressure wave for pipeline leakage localization, Process Safety and Environmental Protection, Vol 104, pp142-149, August 2016, Journal

[19] Wang C. and Yang J., Research on Method for Leakage Detection of Flammable Gas Pipeline, IOP Conference Series, Materials Science and Engineering 394 042077, ACMME, IOP Publishing, 2018, Conference Proceedings

[20] Adnan N.F., Ghazali M.F., Amin F.F., Hamat A.M.A, Leak detection in gas pipeline by acoustic and signal processing, International Conference of Mechanical Engineering Research, IOP Conference Series: Materials Science and Engineering, 100 012013, 2015, Conference Proceedings

[21] Gajbhiye R.N. and Kam S.I., Leak Detection in Subsea Pipeline: A Mechanistic Modelling Approach with Fixed Pressure Boundaries, Offshore Technology Conference, OTC 19347, Texas, 2008, Conference Proceedings

[22] Zhang J., Murphy K., Borchers H., Tornow Stefan and Beushausen Rainer, Transient leak detection in crude oil pipelines, Proceeding of International Pipeline Conference, Calgary Alberta Canada, October 2004, Conference Proceedings

[23] Henrie M., Carpenter P. and Nichlas R.E., Pipeline Leak Detection Handbook, Chapter 6, pp 118120, Gulf Professional Publishing, 2016, Book

[24] Zhang J, Designing a cost effective and reliable pipeline leak detection system. Manchester: REL Instrument Ltd, 1996, Book

[25] Zhang J. and Kane A., Leak Detection and Operations Management In Offshore Pipelines, Proceedings of 2016 International Pipeline Conference, Calgary Alberta Canada, September 2016, Conference Proceedings

[26] Zhang J. and Twomey M., Statistical Pipeline Leak Detection Techniques for All Operating Conditions, 26th Environmental Symposium \& Exhibition, California, March 2000, Conference Proceedings

[27] Farmer J.E., Detecting Leaks in Pipelines, International Society of Automation, Chapter 18, Pp235-239, North Carolina, USA, 2017, Book

[28] Bryce P., Jax P. and Fang J. Special Report: Leak-detection system designed to catch slow leaks in offshore Alaska line, International Pipeline Conference (ASME), Calgary Canada, September 2002, Conference Proceedings

[29] Thodi P., Paulin M., DeGeer D., and Squires M., Real Time Arctic Pipeline Integrity and Leak Monitoring, Arctic Technology Conference, OTC 25604-MS, Houston, February 2015, Conference Proceedings

[30] Oyedeko K.F.K and Balogun H.A., Modeling and Simulation of a Leak Detection for Oil and Gas Pipelines via Transient Model: A Case Study of the Niger Delta, Journal of Energy Technologies and Policy, Volume 5, 2015, Journal

[31] De Sousa A.C. and Romero O.J., Influence of oil leakage in the pressure and flow rate behaviors in pipeline, Latin American Journal of Energy Research, Volume 4, 2017, Journal

[32] Jujuly M., Thodi P., Rahman A., and Khan F., Offshore Arctic Technology Conference, Newfoundland, 2016, Conference Proceedings

[33] Zeng Y., Luo R., Numerical Analysis on Pipeline Leakage Characteristics for Incompressible Flow, Journal of Applied Fluid Mechanics, 12(2):485-494, 2019, Journal

[34] Mansour R.B, Habib M.A., Khalifa A., Toumi-Youcef K.,Chatzigeorgiou D. Computational fluid dynamic simulation of small leaks in water pipelines for direct leak pressure transduction, Computers \& Fluids 57 (2012) 110-123 with Elsevier Publishing, Journal 
[35] Araújo M. V., Neto S.R., Lima A.G.B., and Luna F.D.T., Hydrodynamic Study of Oil Leakage in Pipeline via CFD, Advances in Mechanical Engineering, Article ID 170178, 2014, Journal

[36] Cloete S., Olsen J.E. and Skjetne P., CFD Modelling of plume and free surface behavior resulting from a sub-sea gas release, Applied Ocean Research, Vol 31, 2009, Journal

[37] Zhu H., Lin P., Pan Q., A CFD (computational fluid dynamic) simulation for oil leakage from damaged submarine pipeline, Journal of Energy, Vol 64, 2014, Journal

[38] Igor A. Guz, Marina Menshykova \& Jeom Kee Paik, Thick-walled composite tubes for offshore applications: an example of stress and failure analysis for filament-wound multi-layered pipes, Society of Petroleum Engineers, 2017, Journal

[39] Alexander C. Development of a composite repair system for reinforcing offshore risers, Ph.D. Thesis, Texas A\&M University, College Station, TX, 2007, Journal

[40] Shamsuddoha M, Islam MM, Aravinthan T, Manalo A, Lau K. Effectiveness of using fibrereinforced polymer composites for underwater steel pipeline repairs. Compos Struct, 100:40-54, 2013, Journal

[41] Mally TS, Johnston AL, Chann M, Walker RH, Keller MW. Performance of a carbon-fibre/epoxy composite for the underwater repair of pressure equipment. Compos Struct. 100:542-547, 2013, Journal

[42] CAPP, Canadian Association of Petroleum Producers, Best Management Practice, Use of Reinforced Composite Pipe (Non-Metallic Pipelines), April 2017, Book

[43] Azo Material, Selection Guide for Advanced Composites in Oil and Gas Applications, https://www.azom.com/article.aspx?ArticleID=11254, Date Accessed August 2019, Section of a Web Document

[44] Morozov E and Kuang Y., Design and Analysis of Flexible Reinforced Thermoplastic Pipes (RTPs) for Offshore Oil and Gas Applications, Proceedings of the Twelfth Pacific-Asia Offshore Mechanics Symposium, Gold Coast, Australia, October 2016, Conference Proceedings

[45] Parvez M.D.A, Abdulaziz Y. Asiri, Adel Badghaish, Abdullah K. Al-Dossary, Abdulelah AlMehlisi, Saudi Aramco details nonmetallic products deployment in Oil and Gas, Journal of Oil and 2018, Journal

[46] Zhang J., Atmos International, Client Customer Profile for Royal Dutch Shell, https://atmosi.com/en-us/clients/shell/, Date Accessed 30 September 2019, Section of a Web Document 